Similar examples for a larger number of functions can readily be built.

The following theorem however is true :

If $u_{1}, u_{2}, \cdots, u_{n}$ are single valued functions of the real variable $x$ defined at every point of a certain interval and having at every point of this interval derivatives of the first $n-1$ orders, and if it is possible to strike out the last row and one of the columns of the determinant $D$ in such a way that there is no point of the interval in question at which the remaining determinant and its derivative both vanish, then if $D$ vanishes at every point of the interval, the functions $u_{1}, u_{2}, \cdots, u_{n}$ will be linearly dependent throughout this interval.

This theorem can be readily proved by a slight extension of the method given for instance by Heffter in his book on linear differential equations p. 233.

Ems, Germany,

September 15, 1900.

[Note added November 2, 1900: I have just found in Pascal's book on determinants a reference to three papers by Peano (Mathesis, vol. 9 (1889), p. 75 and p. 110 ; Rend. d. Accad. d. Lincei, ser. 5, vol. 6 (1897), $1^{\circ}$ sem., p. 413), in which the question which I have here considered is taken up. My result is however different from Peano's, which states that the identical vanishing of $D$ is a sufficient condition for linear dependence, provided there is no point at which the first minors corresponding to the elements of the last column all vanish.]

\title{
REPORT ON THE GROUPS OF AN INFINITE ORDER.
}

BY DR. G. A. MILLER.

(Read before Section A of the American Association for the Advancement of Science, New York, June 28, 1900.)

VARIous terms have been employed to designate the smallest elements of which any abstract group is composed. Cayley has called them symbols, * or symbols of operation. Dyck and many others have called them operations $\dagger$ or operators. Frobenius and others have called them elements. I In what follows we shall employ the last one of

\footnotetext{
* Cayley, Phil. Magazine, vol. 7 (1854), p. 41

† Dyck, Math. Annalen, vol. 20 (1882), p. 1.

$\ddagger$ Frobenius, Crelle, vol. 86 (1879), p. 218.
} 
these terms. According to the early definitions of an abstract group a set of distinct elements becomes a group with respect to a given law of combination when it has the property that no new element is obtained by combining the elements of the set in every possible manner according to this law of combination.* In recent years the following additional conditions have been imposed :

(1) The elements must be associative when combined according to the given law. (2) From either of the two equations $s_{1} s_{2}=s_{1}{ }^{\prime} s_{2}, s_{2} s_{1}=s_{2} s_{1}^{\prime}$ it follows that $s_{1}=s_{1}{ }^{\prime}$. (3) If any two of the elements of the equation $s_{1} s_{2}=s_{3}$ are in the given set there is one and only one element in the set which may be used for the third. This definition is much more explicit than Cayley's dictum + "a group is defined by the law of combination of its symbols," since it is easy to give definite laws of combination of a set of distinct elements which do not form a group. Every particular group is, however, defined by the special laws of combination of its symbolsby its own multiplication table.

One of the best known examples of a group of an indefinitely large order is furnished by the totality of finite integers when they are combined by addition. If we denote by $s^{-a}$ the inverse of $s^{\alpha}$, it is clear that the negative exponents of elements may be used to indicate subtraction and that the identical element is $s^{0}=0$, when the elements of a group are combined by addition. In general, if $s$ represents any integer, the exponent of $s$ will indicate the number of times this number is to be added or subtracted. The given group of indefinitely large order is evidently generated by $s_{1}=1$ as well as by $s_{2}=s_{1}^{-1}=-1$, but by no other one of its elements. While $\varphi(m)$ of the elements of any cyclical group of a given finite order $m$ are generators of the group we have here a cyclical group of an indefinitely large order which contains only two generating elements. All the multiples of any integer whose absolute value exceeds unity constitute a subgroup of this group and each one of these subgroups is of an indefinitely large order and involves only two elements that generate it.

In order to obtain a subgroup of a finite order $m$ we may take the complete series of smallest positive residues mod. $m(0,1,2,3, \cdots, m-1)$. When we add any two numbers of this series and use the smallest positive residue in place of the sum we evidently obtain some number of this series.

* Cayley, loc. cit.; Lie, Le centenaire de l'École normale, 1895, p. 486 ;

Klein, Vorlesungen über das Ikosaeder, 1884, p. 5.

† Cayley, Amer. Jour. of Math., vol. 1 (1878), p. 51. 
As the other group conditions are satisfied we have here a cyclical group of order $m$ which is generated by any one of the $\varphi(m)$ numbers that are not greater than $m$ and prime to it. Hence we observe that the finite integers, when combined by addition, furnish very elementary examples of groups of any given finite orders as well as of groups of indefinitely large orders.

Since $x$ cannot be an integer in such an equation as $\delta x=19$, it follows that the integers do not constitute a group with respect to multiplication.* The finite commensurable numbers which exceed zero, on the contrary, constitute such a group. Those which are less than zero do not constitute a group; but, together with those that exceed zero, they clearly constitute a group with respect to the given operation. This group of infinite order is included in the one formed by all the real numbers with the exception of 0 and $\infty$. The latter group is, in turn, included in the abelian group formed by multiplying together all the complex numbers whose absolute value is neither 0 nor $\infty$. The finite commensurable numbers, including 0 , evidently constitute a group of infinite order when they are combined by addition and this group is included in the one formed by all the real finite numbers. The totality of complex numbers of finite absolute value constitute a group, with respect to addition, of which the preceding is a subgroup.

Although all the integers do not form a group with respect to multiplication, yet it is clear that all the powers of any finite integer, with the exception of 0 and 1 , constitute a group of an indefinitely large order with respect to this operation. In order to obtain a group of a finite order by multiplying integers together we may take the $\varphi(m)$ positive numbers which are less than $m$ and prime to it and replace their products by the least positive residues mod. $m$. We thus obtain a very important class of abelian groups which has been studied recently by Weber and others. $\dagger$

While the fundamental operations addition, multiplication, and their inverses furnish such lucid examples of groups of an infinite as well as of a finite order, yet it is not customary to claim that the theory of groups is as old as these elementary operations. At least one group property of these operators must have been observed very early; viz., that the successive application of two elements is equivalent to the single application of some one of them. While

* Weber, Lehrbuch der Algebra, 1899, p. 4 ; of. ibid., 1896, p. 54.

† Weber, Lehrbuch der Algebra, 1899, p. 60. Cf. Burnside, Theory of groups of a finite order, 1897, p. 239. 
this concept is of great theoretic interest yet it did not enter explicitly into the early use of these fundamental operations and hence it may perhaps be said to have played a secondary rôle in the developments along this line.

The concept of group of an infinite order appears in many other early developments but the earliest extensive paper in which this concept occupies a very prominent place seems to be the "Mémoire sur les groupes de mouvements," by Jordan, which was published in the Annali di Matematica, vol. 2 (1868).* No general definition of a group occurs in this memoir but on page 181 a "définition caractéristique d'un groupe de mouvements" is given, which is in accord with the present definition. An abstract of this memoir was published somewhat earlier in the Paris Comptes rendus, vol. 65 (1867). In the beginning of the memoir Jordan points out that the determination of all the groups of movements is equivalent to the determination of all the possible systems of molecules which can be superposed in different positions.

Bravais had studied particular cases of this question, at a much earlier date from the latter point of view, $\dagger$ and applied his results in the study of the structure of crystals. Since Jordan's memoir was inspired by the work of Bravais it might perhaps be said that the theory of groups of an infinite order has its source in the theory of the structure of crystals. However, the group concept does not occupy as prominent a place in the works of Bravais as in the mentioned memoir of Jordan. We proceed to give an elementary exposition of the first part of this memoir with a view to giving an accurate idea of its contents. After some prelimi. nary remarks and a brief study of the composition of movements the author proceeds to determine all the groups of translations in the following manner :

The lengths and the directions of all the translations of the group are represented by line segments starting from a general point $P$. It is first assumed that all of these segments are of finite lengths and the length of the shortest segment $L_{1}$ (or one of the shortest segments, if. there is more than one of minimum length) is represented by $l$. The line through $L_{1}$ is taken for the $x$-axis. An indefinite number of segments of length $l$ are laid off on the $x$-axis starting from $P$. The point $P$ can evidently be transformed into

* Cf. Fricke und Klein, Automorphe Functionen, vol. I., 1897, p. 12. Klein seems to have been the first to make prominent use of the concept of discontinuous groups of an infinite order in function theory; cf. Dyck, Math. Annalen, vol. 20 (1882), p. 2, footnote.

† Bravais, Liouville's Journal, vol. 14 (1849), p. 167. 
any one of these points of division on the $x$-axis by means of some translation of the group. If $P$ could be translated into any other point on the $x$-axis, its distance from $P$ would be $m l \pm r, m$ being an integer and $0<r \leqq l / 2$. If we combine this translation with the translation through $-m l$, which is also in the group, we obtain a translation whose length cannot exceed $l / 2$. As this is contrary to the hypothesis, it follows that the points of division can be transformed into none of the points on the $x$-axis except points of division, by all the translations of the group.

After proving that all the translations parallel to the $x$-axis are generated by a single translation the author proceeds to determine all the possible translations in the plane and proves that all of these are generated by two translations which make a finite angle with each other. This is done as follows: Among the given line segments starting from $P$ we take one $L_{2}$ whose extremity is at a minimum distance from the $x$-axis and represent this distance by $\delta$. The first object is to prove that $\delta$ must be finite. We may assume that the projection $\alpha$ of $L_{2}$ upon the $x$-axis does not exceed $\frac{1}{2} l$ since the group involves translations of length $l$ parallel to the $x$-axis. Since the length of $L_{2}$ cannot be less than $l$, we have

$$
l^{2} \equiv \sqrt{\alpha^{2}+\delta^{2}} \equiv \sqrt{\frac{l^{2}}{4}+\delta^{2}}, \text { or } \delta^{2} \equiv \frac{3}{4} l^{2} .
$$

On the line through $L_{2}$ an indefinite number of segments of the same length as $L_{2}$ are laid off, starting from $P$. Through the points of division lines are drawn parallel to the $x$-axis and through the given points of division of the $x$-axis lines parallel to $L_{2}$ are drawn. The plane is thus divided into parallelograms, as is usually done in the studv of doubly periodic functions. It is then proved that the vertices of these parallelograms can be transformed only among themselves by all the translations of the group and hence there is no finite translation in the plane determined by $L_{1}$ and $L_{2}$ except those which are generated by the translations represented by the segments $L_{1}$ and $L_{2}$. From this it follows that there are two and only two groups of translations, involving only finite translations, in a given plane. In one of these groups all the translations are parallel to a given line; in the other they are generated by two translations making a finite angle with each other.

If a group contains any finite translations in addition to those given above they cannot lie in the plane determined 
by $L_{1}$ and $L_{2}$. We choose one of them, having a minimum projection $\beta$ upon the normal to the given plane, and represent it by $L_{3}$. The length of $\beta$ may be proved to be finite by the following method. The projection of $L_{3}$ upon the given plane may be assumed to lie in any one of the four parallelograms which have $P$ for a common vertex. This parallelogram is so chosen that the distance from the $x$-axis to the terminus of the given projection is $\equiv \delta / 2$. Since $L_{s}$ cannot terminate closer to the $x$-axis than $L_{2}$ it follows that

$$
\delta \equiv \sqrt{\beta^{2}+\frac{\delta^{2}}{4}}, \quad \text { or } \quad \beta^{2} \equiv \frac{3}{4} \delta^{2} .
$$

Space is now divided into parallelepipeds whose vertices are the points into which $P$ may be translated by all the operators of the group generated by the translations represented by $L_{1}, L_{2}, L_{3}$. It is readily proved that a vertex of such a parallelepiped cannot be transformed into any point except a vertex by means of the translations of the group. This is therefore the largest group composed entirely of finite translations in ordinary space and the two groups mentioned above are subgroups of this one. Every point of space which is not a vertex of the given system of parallelepipeds will clearly be transformed into a vertex of a similar system of parallelepipeds by the translations of this group.

There are six groups of translations that involve indefinitely small translations. The first of these is generated by a single infinitesimal translation, the second by an infinitesimal and a finite translation not in the same direction, the third by an infinitesimal and two finite translations the three translations being not co-planar, the fourth is generated by two infinitesimal translations not in the same direction, the fifth by two infinitesimal and one finite translation not in the same plane, and the sixth by three infinitesimal translations not in the same plane. The last of these groups includes all the others as subgroups and each one of these nine groups is of an infinite order.

After the groups which involve only translations have been determined the author proceeds to the groups which are composed of rotations only. Here the matter becomes somewhat more difficult. The first step is to prove that all the axes of rotation must go through the same point. This is done as follows: Let $R_{1}, R_{2}$ be two such rotations. Their combination $R_{1} R_{2}=R_{3}$ is a new rotation around some axis $A_{3}$. Let $x$ be a point on $A_{3}$. $\quad R_{1}$ will bring $x$ to some new point $x_{1}$. Hence the axis of $R_{1}$ must lie on the plane per- 
pendicular to the segment joining $x$ and $x_{1}$ at its middle point. Since $R_{2}$ must transform $x_{1}$ into $x$ its axis must lie in the same plane. Hence the axes of any two rotations must go through the same point. Since this point is transformed into itself by the two rotations $R_{1}, R_{2}$ it must also lie on $A_{3}$. As $A_{3}$ passes through the point $x$, which is not in the plane of the axes of $R_{1}$ and $R_{2}$, the three axes of rotation cannot lie in the same plane. Since any other axis of rotation has to meet each of these three lines it must go through their common point, as the lines are not in the same plane. This proves that all the axes of a group composed entirely of rotations must be concurrent.

It is not difficult to see that there are at least eight groups which are composed of rotations only. The first is generated by the rotation through an angle $2 \pi / n, n$ being any integer. The second is generated by rotating through an infinitesimal angle. The third and fourth are generated by adding to each of the preceding a rotation through $180^{\circ}$ around an axis perpendicular to the given one. The fifth, sixth and seventh are the well known rotations which transform the regular tetrahedron, the cube, and the regular icosahedron $*$ into themselves. These groups are respectively of order 12,24 , and 60 , and may be represented as the alternating group of four letters, the symmetric group of four letters, and the alternating group of five letters respectively. The eighth is composed of all the possible rotations around a point. The proof that there is no other group of rotations is somewhat lengthy, and we shall not enter upon it.

The greater part of the memoir under consideration is devoted to groups which involve both rotations and translations. These have recently been studied by Schönflies, who published his results in two well-known memoirs. $\dagger$ It may be remarked that Jordan's memoir is not entirely free from errors. Sohncke seems to have been the first to observe that an important class of groups was overlooked in the memoirs mentioned. : The determination of all the continuous groups of euclidean and non-euclidean movements in a space of three dimensions was first published by Lie.

Although groups of an infinite order were studied before Lie and Klein began to publish on this subject, yet they were the first to bring them prominently before the pub-

* Cf Klein, Vorlesungen über das Ikosaeder, pp. 1-19.

† Mnth. Annalen, vol., 28, pp. 319-342; vol. 29, pp. 50-80 ; cf. Schönflies, Chicago Mathematical Congress Papers, 1896, p. 341.

† Theorie der Krystallstruktur, 1879, p. 26. 
lic.* Their investigations along this line are so far reaching and so numerous as to deserve a report by themselves. Happily the German Mathematical Association has arranged for a report on Lie's work, which is expected to be presented at the meeting of next year (1901). The report is to be prepared by Engel assisted by Scheffers, Schur, and Kowalewski -men who are in the best possible position to prepare an extensive and reliable report in reference to the work of this great Norwegian mathematician. In view of this fact we shall not consider the works of Lie and his followers in the present report.

In 1882 two remarkable memoirs, dealing with groups of an infinite order, appeared. One of these was published by Dyck in the Mathematische Annalen, volume 20, and the other by Poincaré in the Acta Mathematica, volume 1 . These articles have received so much attention that it seems unnecessary to give an outline of them. Burnside has devoted Chapter XII. of his 'Theory of groups of a finite order (1897) to an exposition of Dyck's memoirs, while many parts of Poincaré's memoir are elucidated in the work on Automorphic Functions by Fricke and Klein, which also appeared in 1897.

This last work makes a clear distinction between continuous groups and groups which contain infinitesimal transformations. It is easy to prove that a continuous group always involves infinitesimal transformations, for if $T$ is any transformation of the group and $T^{\prime}$ the transformation obtained by using parameters which differ by an infinitesimal from those of $T$ then $T^{\prime} T^{-1}$ is infinitesimal. However the existence of infinitesimal transformations does not prove the group continuous. Attention is called $\dagger$ to the fact that in Lie-Engel's work on Transformationsgruppen and in Poincaré's memoir not sufficient emphasis is laid upon this distinction. The work of Fricke and Klein on the group-

* Cf. Klein, Erlangen Programme (1872), Busleterin, vol. 2 (1893), p. 215. Lie's contributions are systematically treated in the three volumes of his Theorie der Transformationsgruppen (1888-93); Differentialgleichungen (1891); Continuierliche Gruppen (1893); Berührungstransformationen, vol. 1 (1896); Klein's Eınleitung in die höhere Geometrie (1893); etc. A considerable part of Klein's work along this line is contained in the two volumes of his Modulfunctionen (1890-92), and in the Automorphe Functionen, vol. 1 (1897). Numerous publications by Picard and Poincaré have also contributed very much towards the general interest in this subject. Picard was the first to develop the theory of linear differential equations parallel with the Galois theory of algebraic equations. Vessiot, Drach, and others have perfected this theory in certain directions.

$\dagger$ Page 65. 
theoretic foundation of automorphic functions is doubtless the best work on discontinuous groups of an infinite order that has yet been published. The direct object of this work is the investigation of the groups of linear substitutions in one variable which do not contain any infinitesimal substitution.

While in Lie's theory the parameters of the product of two transformations are functions of the parameters only of these transformations, Halphen has considered groups in which these parameters are functions both of the variables and of the parameters of the two transformations that are multiplied together. Lie pronounced this group concept trivial and said that it had not found any application.* $\mathrm{He}$ expressed the opinion that the definition of group of transformations had perhaps reached a definite form, but that it was not certain that the general notion of group had as yet assumed such a form.t

Study and Engel seem to have discovered independently $\$$ that the special linear homogeneous group of the plane contains transformations which are not generated by any infinitesimal transformations of the group, as was apparently assumed by Lie.§ Such transformations have been called singular transformations and they have received considerable attention in recent years. The two memoirs by Engel have been followed by numerous other memcirs especially by Taber, || who established the existence of singular transformations in the group of orthogonal substitutions in $n$ variables, $n>3$, and also in the group of linear automophic transformations of a general bilinear form. He has also determined many properties of groups that contain such transformations.

One of his students, Mr. Rettger, has recently investigated $\uparrow$ the two and three parameter subgroups of the general projective group in two variables and of the general linear homogeneous group in three variables with regard to singular transformations, and proved that singular trans-

\footnotetext{
* Theorie der Transformationsgruppen, vol. 3 (1893), p. 19 ; cf. Amer. Jour. of Math. . vol. 11 (1889), p. 182.

† Le centenaire de l'Ecole normale, 1895, p. 486.

$\ddagger$ Engel, Leipziger Berichte, vol. 44 (1892), pp. 277-296 and vol. 45 (1893), pp. $659-696$; cf. BuLLETIN, vol. 3 (1893), p. 66 .

\& Cf. Taber, Bulletin, vol. 6 (1900), p. 199.

I| Taber, Amer. Jour. of Math., vol. 16, p. 130 ; Proc. of the Lond. Mat. Soc., vol. 26 (1895), p. 364; Math. Annalen, vol. 46, p. 561; Bulletin, vol. $6(1900)$, p. 199. Numerous other references are contained in these articles.

I Rettger, Amer. Jour. of Math., vol. 22 (1900), p. 60.
} 
formations occur among the transformations of many of these groups. A list of such subgroups is given at the end of the article. Many of the results of this article are contained in an earlier note by the same author in the Proceedings of the American Academy, vol. 33 (1898), p. 493.

Besides the groups which are explicitly of an infinite order there is a large class of groups whose orders depend upon one or more parameters, which are generally assumed to be finite but may also be regarded as infinitely large. To this class belong the symmetric and the alternating groups of degree $n$,* the cyclical groups of order $n$, the metacyclic and the semi-metacylic groups, of orders $p(p-1)$ and $\frac{1}{2} p(p-1)$ respectively, etc. Such groups are, however, generally classed with the groups of a finite order. We observed above that Burnside devoted a small part of his theory of groups of finite order to the study of groups of an infinite order. In the second volume of Weber's Algebra these groups receive much more attention. Considerable portions of the chapters on linear groups are devoted to considerations which explicitly relate to groups of an infinite order. The object seems to be to devote some space to discussions in which the order is not restricted and then to proceed to the special cases where the order is finite.

The theory of groups of an infinite order is closely related to that of a finite order and the development of the former has been greatly influenced by the latter. The latter, in turn, has contributed very much towards the interest and importance of the former, and, in several instances, it has led to important new developments in this theory ; e.g., Maillet has shown + how the concept of parameter groups, as used by Lie, can be employed in the theory of substitution groups and has made use of this concept to obtain several general theorems on simply isomorphic groups. In a recent article, $\$$ Loewy has established the fundamental theorem that a linear substitution group of infinite order which contains at least one substitution whose characteristic equation has no equal roots, must always contain a substitution whose order exceeds any given number. The presence of such a substitution is therefore a necessary and sufficient condition that the order of a linear group is infinite.

Cornell University, June, 1900

\footnotetext{
* Dyck, Math. Annalen, vol. 22 (1883), p. 72.

† Maillet, Ann. di Matematica, vol 23 (1895), p. 199.

$\ddagger$ Loewy, Math. Annalen, vol. 53 (1900), p. 225.
} 